\title{
Der Katholikentag in Aachen 1986 \\ Befragung von Teilnehmern und Analyse der Berichterstattung
} von Norbert Waldmann

Das Programm des 89. Deutschen Katholikentags in Aachen 1986 umfaßte über 1.000 Einzelveranstaltungen. Hauptgliederungspunkte des Programms waren neben Eucharistiefeiern, Gottesdienst und Gebet, der Arbeitsteil des Katholikentags, der sich in fünf, von den Veranstaltern so benannten, Themensektoren und verschiedene Zentren gliederte. Daneben fanden Begegnungsprogramme - mit Schwerpunkt an einem Tag - und Ausstellungen, wie etwa die Ausstellung „'Heiligtümer' Jugendlicher“, statt. Besonderheiten dieses Katholikentags waren das umfangreiche Wallfahrtsprogramm und die Heiligtumsfahrten, die diesen von früheren unterschieden.

Die Berichterstattung über ein kirchliches Ereignis unterliegt denselben GesetzmäBigkeiten wie die Berichterstattung über andere Ereignisse. Das bedeutet, daß bei der Berichterstattung eine Auswahl stattfinden muß. Dabei ist nicht das wichtigste, daß dies geschieht, sondern wie dies geschieht. Keine Berichterstattung kann umfassend oder vollständig sein. Es ist auch nicht gewünscht, denn Ereignisse werden erst dann vermittelbar, wenn sie strukturiert werden und dadurch Komplexität genommen wird.' Schließlich ist jede Wahrnehmung immer schon eine Interpretation der Realität. Ein Vergleich mit dem „Ereignis selbst“ ist ausgeschlossen.

Trotzdem gibt es immer wieder Diskussionen über die „Richtigkeit“ der Berichterstattung über Ereignisse, Personen, Themen in den Medien. Der Wunsch, sich hierüber Klarheit zu verschaffen, ist verständlich, denn Medien haben wesentliche Vermittlungsleistungen übernommen. ${ }^{2}$ Vieles von dem, was in der Welt geschieht, würden wir gar nicht erst zur Kenntnis nehmen, gäbe es die Medien nicht.

Durch die Selektionsmechanismen suggerieren die Medien uns aber auch, daß das, was sie berichten, zudem wichtig ist. Sie geben uns eine „Hit-Liste“ der Ereignisse vor. Die Prioritäten, die dabei gesetzt werden, sind jedoch nur eine Seite des Vermittlungsaspektes. Tiefergehend ist die andere Seite: es wird nicht nur vorstrukturiert, was wichtig ist, sondern auch, was daran wichtig ist. Kurzum, Medien können auch bestimmen, wie wir über Ereignisse, Personen und Themen zu denken haben.

Wenn es nun so ist, daß Realität nie vollständig erfaßbar ist, weder durch Medien noch durch eigene persönliche Wahrnehmung, so läßt sich die Frage nach der "Richtigkeit“ medialer Vermittlungsleistungen nicht an einem absoluten Nullpunkt messen. Dies führt dazu, daß die Berichterstattung über ein Ereignis nicht an einem Kriterium gemessen werden kann, sondern sie kann immer nur im Vergleich beurteilt werden.

Die vergleichende Analyse der Berichterstattung von Fernsehen und Tageszeitungen zum Katholikentag ist deshalb das Thema dieser Untersuchung ${ }^{3}$, aus der hier einige zusammenfassende Befunde vorgestellt werden. Dieser Vergleich bezieht auch eine Befragung der Teilnehmer und deren Wahrnehmung des Ereignisses ein. ${ }^{4}$ Es kann daher nicht nur Auskunft gegeben werden, wie über ein Ereignis berichtet wird, sondern auch darüber, wie vielschichtig Ereignisse wahrgenommen werden. Die verschiedenen Facetten eines Ereignisses in der Wahrnehmung der Teilnehmer und der darüber berichterstattenden Medien Presse und Fernsehen sind der Kern der Untersuchung.

Dr. Norbert Waldmann M.A. ist wissenschaftlicher Referent des Haus-Bredow-Institutes für Rundfunk und Fernsehen an der Universität Hamburg. 


\section{Vorgehensweise}

Mit der Methode der Inhaltsanalyse $e^{5}$ untersucht wurde

- die Berichterstattung in vier überregionalen Tageszeitungen,"

- die WDR-Fernsehberichterstattung,

- das Programmheft des Katholikentags,

- Antworten auf offene Fragen bei der Befragung von Teilnehmern des Katholikentags.

Auf der Folie des Programms des Katholikentags wurde ein Kategorienschema entwickelt, das für das Programm selbst, für die Berichterstattung in den Zeitungen, für die Berichterstattung im Fernsehen und für die Verschlüsselung der in der Umfrage offen gestellten Fragen dient.

Die Analyse der Zeitungen umfaßte jeweils die gesamte Zeitungsausgabe für den Zeitraum vom 1. bis 20.9.1986, also drei Wochen lang. Da der Katholikentag vom 10.9. bis 14.9. stattfand, bestand so die Möglichkeit, auch die Voraus- und die Nachberichterstattung zu erfassen. Ferner wurde die während des Katholikentgs an vier Tagen erschienene „Offizielle Zeitung zum 89. Deutschen Katholikentag, Aachen“ in die Untersuchung einbezogen.

Von der Fernsehberichterstattung wurden alle Sendungen berücksichtigt, die sich mit dem Katholikentag beschäftigten und innerhalb der ARD oder im WDR-Regionalprogramm oder im WDR III ausgestrahlt wurden. Sendungen des ZDF wurden nicht berücksichtigt. Da zwischen ARD und ZDF eine Arbeitsteilung bestand, ist zu vermuten, daß sich das Sendevolumen zwar nicht verdoppelt hätte, wohl aber wegen der Regionalberichterstattung um ein Drittel erhöht hätte.

Alle Fernsehbeiträge stammen aus der Zeit vom 5.9. bis 16.9 .86 und wurden vom WDR für die Analyse bereitgestellt. ${ }^{8}$ Die Dauer aller in die Analyse einbezogenen Fernsehsendungen betrug fast 8 Stunden und setzte sich zusammen aus Nachrichtenund Magazinbeiträgen und Sondersendungen. Letztere machten darunter einen Anteil von $61 \%$ aus.

Die Fernsehbeiträge und Zeitungsartikel wurden bei der Inhaltsanalyse genauer aufgegliedert zu von uns definierten Informationseinheiten. Dies ist deshalb notwendig, da sich die Journalisten in ihren Beiträgen häufig auf mehrere Inhalte oder Veranstaltungen beziehen. Als Beispiel seien hier Überblicksartikel erwähnt, die die Ereignisse eines Tages Revue passieren lassen. Bei den Zeitungen waren diese analytischen Einheiten in der Regel identisch mit einem Absatz, ${ }^{9}$ beim Fernsehen mit einer Sequenz. ${ }^{10}$

Die zentralen Kategorien geben Auskunft über die Fragen, welche Veranstaltungen und Programminhalte erwähnt werden. Dabei wird nicht nur die Häufigkeit, sondern auch der Umfang der Informationseinheit, in der die Nennung stattfindet, erfaßt.

Die Analyse des Umfangs und des Inhalts der Berichterstattung bietet jedoch nur begrenzt die Möglichkeit der Justierung. Wie facettenreich ein Ereignis ist, wie Wahrnehmung begrenzt ist, läßt sich damit unter $U$ mständen allenfalls für die Journalisten sagen. Auf diese Weise aber ist keine Aussage möglich, ob dieses Ereignis auch so von den Teilnehmern wahrgenommen wurde. Wie der Katholikentag von Teilnehmern 
gesehen wurde, wurde deshalb mit einer postalischen Befragung unter fest angemeldeten Teilnehmern des Katholikentags ermittelt. Damit ist ein Vergleich zwischen der Berichterstattung und der Wahrnehmung der Teilnehmer möglich.

Für die Befragung wurde aus den Besuchern, die sich fest für einen der Themensektoren aus dem Veranstaltungsprogramm des Katholikentages oder zu einer mit dem Katholikentag in Verbindung stehenden Wallfahrt angemeldet hatten, eine Zufallstichprobe von 304 Personen gezogen. Die Basis für die Stichprobenziehungen bildeten 39.800 Teilnehmer. ${ }^{12}$ Aus der „Gästeliste“ des Zentralkomitees der deutschen Katholiken wurden ebenfalls nach Zufallsprinzip 40 Adressen ausgewählt. Von den angemeldeten, angeschriebenen Teilnehmern sandten $77 \%$ ihren Fragebogen zurück, so daß sich die Auswertung unter Einbezug der offiziellen Gäste auf insgesamt 233 Antworten stützen kann. ${ }^{13}$

Der Fragebogen enthielt Fragen, die dazu dienten, die subjektiv wahrgenommene Wichtigkeit, etwa durch Teilnahme an einzelnen Veranstaltungen, die Erinnerung an Inhalte und Einzelereignisse des Katholikentags zu erfassen. Die Fragen wurden auf der Grundlage der vorliegenden Programmbroschüre und weiterer Vorabinformationen zum Katholikentag entwickelt.

\section{Besonderheiten}

\subsection{Die Teilnehmer}

$\mathrm{Zu}$ dem Katholikentag kamen Besucher aus der ganzen Bundesrepublik. Den Schwerpunkt bildete die Region, in der der Katholikentag stattfand, das Bundesland Nordrhein-Westfalen. ${ }^{14}$ Die Diaspora im Norden der Bundesrepublik war am schwächsten vertreten.

Jeweils gut die Hälfte der Befragten hat an der Eröffnungskundgebung am Mittwoch und am Hauptgottesdienst am Sonntag teilgenommen. Neben den Gelegenheiten zur Verehrung Gottes in Eucharistie und Sakrament wurden die Möglichkeiten der Begegnung am intensivsten genutzt. Sie waren auch für die Mehrheit der Teilnehmer das zentrale Erlebnis. Es schätzte deshalb auch die Mehrheit der Befragten (52\%) eher die Begegnungen mit Mitchristen und Einwohnern Aachens eindrucksvoller ein als die Begegnung mit Hohen Würdenträgern der Kirche (9\%), Politikern (3\%) oder anderen prominenten Persönlichkeiten (3\%).

Wie die jeweiligen Angebote genutzt werden, hängt von der Interessenlage ab, die bereits ausschlaggebend für die Entscheidung zu der Anmeldung für einen Themensektor bzw. zur Wallfahrt war. Davon abhängig ist auch die weitere Einschätzung des Ereignisses aus der Erinnerung.

Unterschiedlich aufgefaßt wurde die Wahl des Veranstaltungsortes, dessen besonderer Wert rund einem Viertel der Befragten verborgen blieb oder im Ärger über organisatorische Unzulänglichkeit unterging. Betont werden muß aber, daß drei Viertel der Befragten mit der Organisation „sehr zufrieden“ oder "zufrieden“ waren. Verständnisschwierigkeiten mit dem Motto des Katholikentags und der einzelnen Programmtage hatte ebenfalls rund ein Viertel. Einen Bezug zwischen Themen und Veranstaltungen konnten die meisten bei dem Leitwort zum Katholikentag „Dein Reich komme" erkennen. Am schwierigsten war der Bezug zu dem Motto „die Zukunft ist schon geboren“. 
Der „Katholikentag von unten “ war für ein Drittel der Besucher ein fester Bestandteil ihrer Aktivitäten. Diejenigen, die ihn besuchten, empfanden ihn auch als Ergänzung des offiziellen Katholikentags. Da in der Tat dort Themen behandelt wurden, die auch für einige Befragte auf dem offiziellen Katholikentag nicht intensiv genug behandelt wurden, kommt dem „Katholikentag von unten“ diese Enrichmentfunktion zu.

Die während des Katholikentags aufgetretene Diskussion um die Teilnahme von Politikern der Partei Die Grünen spiegelt sich auch in einigen Antworten wider. Für $9 \%$ der Befragten war es „das Wichtigste und Vordringlichste“, um das es bei diesem Katholikentag ging. Generell betrachten die Befragten den Katholikentag nicht als politikfreien Raum, denn lediglich $7 \%$ sind der Auffassung, daß „besser keine Politiker teilnehmen sollten“.

\subsection{Die überregionalen Tageszeitungen}

Nicht nur die Besucher wurden durch den Katholikentag gefordert - sie mußten aus über 1.000 verschiedenen Programmpunkten eine Auswahl treffen - sondern auch die darüber berichtende Presse.

Sie trug dem 89. Deutschen Katholikentag mit 76 Artikeln in vielfältiger Weise Rechnung und berichtete darüber in Wort und Bild sowie in allen ihr zur Verfügung stehenden Formen der Berichterstattung. So wurde auch dieses Ereignis in den Kommentarspalten entsprechend gewürdigt.

Der außerordentlichen Vielzahl und Vielfalt der Veranstaltungen versuchte die Presse dadurch gerecht zu werden, daß sie häufig einen generellen Überblick über Veranstaltungen und Inhalte gab, ohne jedoch auf einzelne im Detail einzugehen. Ausführlicher gingen die zentralen Großkundgebungen $\left(24 \%\right.$ Anteil am Gesamtumfang ${ }^{15}$ der Berichterstattung) und die Veranstaltungen des Arbeitsteils des Katholikentags (17\%) in die Veröffentlichungen ein. Weniger präsent waren andere Programmpunkte des Katholikentags, wie Begegnungs- und Kulturprogramme, aber auch Eucharistiefeier und Heiligtumsfahrt. Inhaltlich konzentrierte sich die Berichterstattung auf die Veranstaltungsinhalte, aber auch auf Diskussionsthemen, die auf dem Katholikentag entstanden waren. Zielsetzungen des Katholikentags, wie zum Beispiel der Diskussion über den "Schutz des ungeborenen Lebens" (11 \%) und „Neue Technologien, Umweltschutz, Kernenergie" (9\%) wurde entsprechend Rechnung getragen. Ausführlich wurde aber auch über die kontrovers geführte Diskussion der Teilnahme von Politikern der Partei Die Grünen und deren Politik berichtet (13\%).

Bemerkenswert an der Presseberichterstattung ist, daß der Katholikentag die Printmedien für kirchliche und religiöse Themen sensibilisierte. Im Vorfeld, während und nach dem Katholikentag erschienen eine Reihe von Beiträgen, die zwar den Katholikentag erwähnten, ihn aber nicht selbst zum Hauptpunkt der Berichterstattung machten (13\% Anteil an der Gesamtberichterstattung).

\subsection{Die Katholikentagszeitung}

Während des Katholikentags erschien an vier Tagen die „Offizielle Zeitung zum 89. Deutschen Katholikentag, Aachen“, die „Katholikentagszeitung“. Die Katholikentagszeitung griff in ihren vier Ausgaben ein breites Spektrum von Veranstaltungen und Themen des Katholikentags auf. In ihr kamen sowohl „Durchschnittsteilnehmer" als auch Teilnehmer auf den Podien, insbesondere die Geistlichkeit, zu Wort. In den 
vier Zeitungsausgaben wurden beinahe $500 \mathrm{Namen}$ von Personen genannt bzw. direkt oder indirekt zitiert.

Die Analyse fördert drei Ebenen der Berichterstattung zu Tage: Ergänzend zu den Inhalten des Katholikentags wurden durch Beiträge des Klerus bestimmte Themen hervorgehoben. Diese Beiträge sollten nicht nur kritisch Inhalte reflektieren, sondern dienten auch der Stärkung des Glaubensbekenntnisses. Die Katholikentagszeitung bildete ferner in breiter Weise den Katholikentag ab. Die Artikel beziehen sich dabei sowohl auf bereits stattgefundene Veranstaltungen als auch auf noch stattfindende Veranstaltungen. Damit übernahm die Katholikentagszeitung unter Umständen Steuerungsfunktionen für die persönliche Gestaltung des Programmablaufs einzelner Teilnehmer.

Letztlich kommt der Katholikentagszeitung noch eine dokumentierende Funktion zu. Der Teilnehmer, der sich diese Zeitung kaufte, konnte zuhause nochmals das Geschehen Revue passieren lassen; in Verbindung mit selbst Erlebtem und nunmehr noch Hinzugelesenem geistig das Ereignis verarbeiten. Er konnte sich - und hierfür spricht beispielsweise der umfangreiche Bildteil mit insgesamt 200 Bildern - selbst wieder finden, konnte anderen mit der Katholikentagszeitung dokumentieren, wie es war und wo er gewesen ist. Sie ist somit auch ein Erinnerungsstück.

\subsection{Die Fernsehberichterstattung des WDR}

Beide öffentlich-rechtlichen Rundfunkanstalten, ARD und ZDF, sowie die Dritten Programme berücksichtigen den Katholikentag in ihrem Programm. Dabei war für die ARD der Westdeutsche Rundfunk verantwortlich, da in seinem Sendegebiet der Katholikentag 1986 stattfand. Entsprechend fand das Ereignis Eingang in die Sendungen des III. Programms des Westdeutschen Fernsehens, sowie in die Vorabend-Regionalberichterstattung innerhalb des Gemeinschaftsprogramms der ARD. Exemplarisch für die Fernsehberichterstattung wurden hier die vom Westdeutschen Rundfunk zu verantwortenden Sendungen untersucht. Beiträge zum Katholikentag wurden sowohl in den aktuellen Nachrichten- und Magazinsendungen als auch in Kirchen- und Sondersendungen ausgestrahlt. Insgesamt betrug die Zahl der Beiträge 30.

Das Fernsehen legte - mit 164 Sendeminuten - einen Schwerpunkt auf die zentralen Kundgebungen, alleine schon bedingt durch die Live-Berichterstattung von der „Europa-Kundgebung" und Hauptkundgebung. Bezieht man die hier nicht nicht zur Untersuchung anstehende Übertragung des Abschlußgottesdienstes durch das ZDF mit ein, so liegt auch ein weiterer Schwerpunkt auf der Eucharistiefeier. Einem besonderen Anliegen des Aachener Katholikentags, den Wallfahrten, trug das Fernsehen in der Reihe "Gott und die Welt" Rechnung. Starken Bezug zum Katholikentag hatte ein persönliches Bekenntnis des Glaubens vermittelnd - das „Wort zum Sonntag", das entgegen der üblichen Dramaturgie vom Aachener Marktplatz aus gesendet und vom Ortsbisch of Dr. Klaus Hemmerle gesprochen wurde.

Neben diesen Veranstaltungen und Inhalten war jedoch das Spektrum weitaus größer. Eine Ausweitung kam vor allem durch den Versuch der Phone-in-Sendung (Freitag, 12.9.) zustande. Hier stellten die Anrufer Fragen, die zum Teil nicht mit Veranstaltungen des Katholikentags in Verbindung zu bringen waren, zum Teil Inhalte berührten, die bisher nicht oder auch gar nicht auf dem Katholikentag gefragt waren. 
Ferner war auch die Phone-in-Sendung nicht nur auf Frage und Antwort reduziert, sondern enthielt einen Überblick zu Beginn, Musikeinlagen und eine Fragerunde mit im Studio anwesenden Jugendlichen. Bandbreite wurde dadurch erreicht, daß auch die Live-Sendung der Hauptkundgebung sich nicht nur auf die Übertragung der Reden beschränkte.

Veranstaltungen, die nicht mit dem offiziellen Programm des Katholikentags in Einklang zu bringen waren, wurden angesprochen, jedoch in geringem Umfang. Es waren dies der „Katholikentag von unten" und im Nachgang zum Katholikentag eine Wallfahrt verheirateter Priester. Im Zusammenhang mit dem „Katholikentag von unten" stand auch die Diskussion um die Teilnahme der Partei Die Grünen. Sie fand im Ausmaß ebenfalls begrenzt Eingang in die Berichterstattung.

Wie auch in der Presse zu lesen, waren im Fernsehen am häufigsten Politiker zu sehen, wurden mit dem Namen erwähnt oder kamen auch zu Wort. $43 \%$ der Informationseinheiten zeigten Politiker. In einem Drittel aller Informationseinheiten war der Klerus vertreten, $29 \%$ hohe geistliche Würdenträger und zu $3 \%$ Geistliche, Priester und Ordensmitglieder. Weitere $15 \%$ der Informationseinheiten enthielten Personen, die auch im Programmheft des Katholikentags als Akteure auf den Podien und in den Veranstaltungen aufzufinden waren, sich aber nicht - weder politisch noch klerikal näher zuordnen ließen.

Dem Fernsehzuschauer werden besonders in Erinnerung bleiben die Teilnehmer an der Diskussionssendung, Professor Dr. Hans Maier, Bischof Dr. Karl Lehmann und Dr. Marita Estor. Die Letztgenannten waren nahezu nur dort im Fernsehen in Erscheinung getreten. Professor Dr. Hans Maier hingegen war auch in den Nachrichtensendungen im Bild zu sehen und zu hören. Der herausgehobenen Position entsprechend war der Vorsitzende der deutschen Bischofskonferenz Kardinal Joseph Höffner, ebenfalls sowohl in Einzelsendungen, insbesondere Live-Übertragungen, Magazin-Sendungen, als auch in Nachrichtensendungen präsent. In geringem Umfang traf dies auch auf den Bischof von Aachen, Klaus Hemmerle, zu.

Neben Einzelpersonen traten im Fernsehen vor allen anderen eine Gruppe Teilnehmer in Erscheinung: die Jugendlichen. Ähnlich wie in der Sendung "Gott und die Welt" den Wallfahrten als einer Besonderheit Rechnung getragen wurde, so wurden sie nicht nur in dieser Sendung, sondern auch in anderen häufig gezeigt und/oder kamen zu Wort.

Hierzu ist vor allem noch die Sendung „Hier und heute unterwegs“ zu zählen. Auch die oft eingeblendeten Musikpassagen vermittelten ein jugendliches Bild vom Katholikentag. Bei einem überwiegenden Anteil an jugendlichen Besuchern ist dies gerechtfertigt, wie auch die Tatsache, daß neben den "Experten “ in der Phone-in-Sendung Jugendliche ins Studio eingeladen waren und ihre Eindrücke mitteilten.

\section{Ein Ereignis - Viele Perspektiven}

Der Katholikentag in Aachen war für jeden der Teilnehmer ein individuelles Ereignis. Dies gilt für die 100.000 Besucher ebenso wie für die dort arbeitenden Journalisten. Wenn hier von den Teilnehmern und den Journalisten die Rede ist, so lediglich als kontrastierende Gruppen. In Wirklichkeit gibt es letztlich nicht den Besucher und den Journalisten. Besucher unterschieden sich voneinander, Journalisten auch. 
Für die Besucher war der Katholikentag in erster Linie ein Ort der Begegnung. 34 \% der befragten Teilnehmer hielten dies für das Wichtigste und Vordringlichste, um das es bei diesem Katholikentag ging. Begegnung beinhaltet dabei das gesamte Bedeutungsfeld und ist Zusammenkunft, Wiedersehen, Beisammensein und Gemeinschaft zugleich. Die von den Veranstaltern angebotenen Anlässe hierfür erscheinen beinahe beliebig. So diente für die Teilnehmer auch der die meisten Programmpunkte umfassende Arbeitsteil, den $76 \%$ für ,sehr wichtig “ oder „wichtig“ hielten, nicht nur oder gar weniger als Arbeitsstätte, sondern als Treffpunkt Gleichgesinnter. Ähnlich sind auch die spirituellen Veranstaltungen wie Eucharistie, Gebet und Meditation anzusehen, denen $83 \%$ die gleiche Wichtigkeit einräumten.

Diese beiden Programmpunkte, die Möglichkeiten der engen Begegnung boten, erfüllten denn mehr diese Funktion, als die großen, mit stattlicher Teilnehmerzahl und geistlicher und weltlicher Prominenz ausgestatteten Kundgebungen. Die große Ansammlung von Menschen schafft alleine noch nicht das Gefühl der Gemeinsamkeit und Gemeinschaft. In der Menschenmasse bleibt der einzelne oft isoliert.

Für die auf dem Katholikentag und an dem Ereignis arbeitenden Journalisten ist solches nur schwer medial aufbereitbar. Immerhin haben mit Bildern arbeitende Medien hier noch eher Möglichkeiten des Ausdrucks als Medien, die sich auf das Wort beschränken.

Es ist jedoch nicht nur die Begrenztheit in der Darstellungsweise. Es ist auch ganz einfach die Tatsache, daß Journalisten in erster Linie wegen ihrer Arbeit an dem Katholikentag anwesend sind. Dadurch folgt die Sichtweise eines Ereignisses einem professionellen Raster, das selbstverständlich verschieden ist von dem der Teilnehmer. Der gleiche Begriff bekommt dabei mitunter eine andere Bedeutung. So registriert der Journalist ebenfalls beispielsweise „Begegnung“, aber es ist für ihn ein Programmpunkt unter vielen, und es ist zunächst einmal derjenige, unter dem er im ausgedruckten Programmheft angekündigt ist. Es wird erst daraus ein berichtenswertes Ereignis, entspricht es seinen professionellen Nachrichtenkriterien wie Prominenz, Anzahl der Teilnehmer und anderem.

Jedoch auch die Journalisten sind diversen Teilgruppen zuzuordnen, und dabei ist nicht in erster Linie die Tatsache ausschlaggebend, ob der Journalist für Printmedien oder für audiovisuelle Medien arbeitet. Die Analyse zeigt, daß die „Katholikentagszeitung" eine anders geartete Berichterstttung über den Katholikentag aufwies als die überregionalen Tageszeitungen und auch das Fernsehen.

Schließlich hat die konfessionelle Publizistik eine spezielle Zielgruppe, und sie spiegelt auch wohl eher die Meinungen innerhalb des Katholizismus wider, wie sie auch Forum für innerkirchliche Diskussion ist, als dies in der übrigen Publizistik der Fall ist. Dies setzt bei den Lesern Vorwissen und bei den Journalisten tiefergehende Kenntnis der Materie voraus.

Wie unterscheiden sich nun die Perspektiven? Nehmen wir die Daten aus den Inhaltsanalysen und der Umfrage und vergleichen sie. Uns interessiert dabei vor allem, welche Veranstaltungen in den Vordergrund gerïckt wurden und welche Inhalte wesentlich waren. Bei diesem Vergleich soll aber auch noch eine weitere Ebene hinzugefügt werden, die des Veranstalters, die sich in seinem Programm ausdrückt. Verständlich ist, daß hierbei sowohl Veranstaltungen wie der "Katholikentg von unten“ oder In- 
halte wie die „Politik der Partei Die Grünen und Kirche“ keinen Eingang gefunden haben. Diese Punkte fallen zwar nicht für einen Vergleich gänzlich aus, aber markieren bereits, daß das Programm eine andere Position setzen muß.

Ein solcher Vergleich kann durch die unterschiedlichen Erhebungen - Umfrage, Analyse von Zeitungen, Analyse von Fernsehsendungen - nur recht holzschnitthaft sein. Wir beschränken uns darauf, die Rangplätze miteinander zu vergleichen, die auf den jeweiligen Prozentzahlen basieren. Rang 1 nach dem Programm nehmen die Veranstaltungen im Programmheft ein, die dem Arbeitsteil des Katholikentags zuzurechnen sind. Auch in der „Katholikentagszeitung“ wird am häufigsten darüber berichtet. In der spontanen Erinnerung der Teilnehmer allerdings nehmen sie lediglich Platz 2 ein, die untersuchten Tageszeitungen weisen dem Arbeitsteil Platz 3 zu, und noch weniger Stellenwert räumt diesem das Fernsehen ein.

Die Diskrepanz zwischen Programm und Umfrage sowie Tageszeitung erklärt sich daraus, daß dort die isolierte Betrachtung einer einzelnen Veranstaltung hinter der Gesamtsicht des Katholikentags zurücksteht. Beim Fernsehen nehmen die zentralen Kundgebungen unbestreitbar den ersten Platz ein. Wir erklärten dies zuvor bereits durch die beiden Live-Übertragungen von der "Europa-Kundgebung" und der "Hauptkundgebung " aber auch durch die Originalmitschnitte in anderen Sendungen. Damit nähert sich auch dieses Medium dem Stellenwert, den die Veranstalter den zentralen Kundgebungen einräumen, denn hier greift die Auswertung des Programmheftes zu kurz. Zentrale Kundgebungen sind naturgemäß Massenveranstaltungen, die jedoch, um solche zu sein, dosiert angeboten werden müssen.

Am zweithäufigsten ließen sich Veranstaltungen im Programm des Katholigentags als Begegnungsprogramm einstufen. Sie fanden bei befragten Teilnehmern großen $\mathrm{Zu}-$ spruch und waren auch in der „Kirchentagszeitung “ häufig auszumachen. Die beiden anderen Medien bezogen sich weniger darauf, am seltensten noch Tageszeitungen.

Es zeigt sich insgesamt, daß sowohl die befragten Teilnehmer, als auch die inhaltsanalytisch untersuchten Medien nicht die Prioritäten setzen, wie die Programmbroschüre zum Katholikentag dies nach den dort angebotenen Veranstaltungen vielleicht erwarten läßt. Am weitesten davon „entfernt" haben sich die untersuchten Tageszeitungen, am dichtesten dabei liegt die „Katholikentagszeitung“. Diese stimmt auch in der Berücksichtigung der Veranstaltungen am besten noch mit den Teilnehmern überein. Die Tageszeitungen und das Fernsehen folgen hier einer anderen Auswahl.

Unter den Medien aber wiederum nimmt die „Katholikentagszeitung“ eine Sonderstellung ein. Die hierfür arbeitenden Journalisten können für sich in Anspruch nehmen, für die konfessionelle Presse zu arbeiten. Tageszeitungen und Fernsehen als weltliche Medien stimmen in ihren Beiträgen, was die Berücksichtigung bestimmter Veranstaltungen anbetrifft, relativ gut überein.

Wir wollen beim nachfolgenden Vergleich (Tabelle 1) auch die Inhalte der Veranstaltungen berücksichtigen. Es soll ebenfalls das ausgedruckte Programm des Katholikentags einbezogen werden. Allerdings bringt hier eine Zuordnung nach vorgegebenen Kategorien Schwierigkeiten, da die Ankündigungen verständlicherweise knapp gehalten sind. Die auf diese Weise gewonnenen inhaltsanalytischen Daten können folglich nur grobe Anhaltspunkte liefern. Erinnert werden muß aber auch daran, daß auf eine ähnliche Weise der Besucher sich aus der Programmbroschüre Informationen beschaffte. 
Tabelle 1

Bedeutung der Veranstaltungen des Katholikentags bei Medien, Teilnebmern und Programm

Vergleich inhaltsanalytischer und Befragungsdaten*)

\begin{tabular}{|c|c|c|c|c|c|}
\hline & $\begin{array}{l}\text { Pro- } \\
\text { gramm } \\
\text { Rang }\end{array}$ & $\begin{array}{l}\text { Teil- } \\
\text { nehmer } \\
\text { Rang }\end{array}$ & $\begin{array}{l}\text { Tages- } \\
\text { zeitung } \\
\text { Rang }\end{array}$ & $\begin{array}{l}\text { Katholiken- } \\
\text { tagszeitung } \\
\text { Rang }\end{array}$ & $\begin{array}{l}\text { Fern- } \\
\text { sehen } \\
\text { Rang }\end{array}$ \\
\hline Arbeitsteil & 1 & 2 & 3 & 1 & 5,5 \\
\hline Begegnungsprogramme & 2 & 3 & 8 & 2 & 7 \\
\hline Kulturprogramme & 3 & 9,5 & 9,5 & 8,5 & 5,5 \\
\hline $\begin{array}{l}\text { Eucharistiefeier, Gebete; } \\
\text { Meditationen }\end{array}$ & 4 & 4 & 7 & 6 & 9,5 \\
\hline Zentren & 5 & 9,5 & 9,5 & 7 & 9,5 \\
\hline $\begin{array}{l}\text { Wallfahrten; Heiligtums- } \\
\text { fahrten }\end{array}$ & 6 & 5 & 6 & 8,5 & 4 \\
\hline Zentrale Kundgebungen & 7 & 7,5 & 2 & 3 & 1 \\
\hline $\begin{array}{l}\text { Katholikentag als Gesamtver- } \\
\text { anstaltung }\end{array}$ & 9 & 1 & 1 & 4 & 3 \\
\hline „Katholikentag von unten“ & 9 & 7,5 & 4 & 10 & 8 \\
\hline $\begin{array}{l}\text { Kein konkreter Bezug zu } \\
\text { Veranstaltungen erkannbar }\end{array}$ & 9 & 6 & 5 & 5 & 2 \\
\hline
\end{tabular}

*) Bei den untersuchten Medien wurde jeweils zur Ermittlung der Rangplätze der Anteil am Gesamtumfang bzw. an der Gesamtdauer herangezogen. Die Rangplätze der Teilnehmer stützen sich auf die Antworten auf die Frage, was das "Wichtigste und Vordringlichste" auf diesem Katholikentag war. Die Daten werden zusammengefaßt wiedergegeben.

Nach dieser Analyse liegen zwei Inhalte nahezu gleichauf mit jeweils $20 \%$ bzw. $21 \%$. Es sind dies Glaubensverkündigung, Glaubensbekenntnis und Sakrament und damit die zentralen Anliegen des Katholikentags. Ein weiterer Schwerpunkt ist im kulturellen Bereich auszumachen. Dies wird bewirkt durch die vielen Ankündigungen von "Geistlichen Mittags- und Abendkonzerten“, das gemeinsame Singen in den Themensektoren, aber auch durch das umfangreiche, alle Bereiche der Kultur umfassende, in der Programmbroschüre ausgewiesene „Kulturprogramm“. So gehören hierzu einzelne Vortragsveranstaltungen ebenso wie Ausstellungen anläßlich des Katholikentags und der musikalische Teil des „Programms unter freiem Himmel“.

Kulturelle Inhalte stellten zwar einen wichtigen Schwerpunkt des Katholikentags dar, wurden aber vermutlich häufig von den Teilnehmern unter dem Aspekt der „Begegnung" gesehen, zweifelsohne eine Funktion und damit ein Inhalt, den diese Veran- 
staltungen bestens erfüllten. Dies erklärt auch, warum dieser Bereich in der spontanen Nennung von Inhalten bei den Teilnehmern kaum eine Rolle spielt.

Da diese Inhalte medial nur schwer vermittelbar sind, werden auch die kulturellen Aspekte in den Medien nachrangig behandelt. Dies gilt insbesondere für die untersuchten Tageszeitungen und für die „Katholikentagszeitung“, das Fernsehen widmet sich diesem Bereich stärker.

Auch die sakralen Themen sind im Fernsehen umfangreicher präsent als in den PrintMedien. Es nähert sich hier überdies am ehesten den befragten Teilnehmern. Dieser Bereich nimmt unter den genannten Inhalten den gleichen Rangplatz mit den im Fernsehen berichteten Inhalten ein.

Insgesamt gesehen decken sich bei diesem Vergleich die von den befragten Teilnehmern bevorzugten Inhalte noch am ehesten mit den durch das Programm angebotenen. Das ist allerdings selbstverständlich. Bemerkenswerter ist, daß jedoch in Teilbereichen Abweichungen bestehen.

So darf es denn nicht verwundern, wenn auch die Medien sich von dem einmal geplanten Programm entfernen. Dies gilt auch für die „Katholikentagszeitung“, aber ganz besonders für die untersuchten Tageszeitungen. Die vorliegenden Daten legen eine Abstufung nach der Distanz zu den Schwerpunkten des Programms nahe. Die erste Stufe der Distanz findet sich bei den Teilnehmern, die zweite bei der „Katholikentagszeitung", die dritte bei der Fernsehberichterstattung und die vierte und letzte Stufe bei den untersuchten Tageszeitungen. Dies in den Daten vorgefundene Struktur bleibt auch erhalten, vergleicht man die Medien untereinander.

Tabelle 1

Bedeutung der Veranstaltungen des Katholikentags bei Medien, Teilnebmern und Programm

Vergleich inhaltsanalytischer und Befragungsdaten*)

\begin{tabular}{|c|c|c|c|c|c|}
\hline & $\begin{array}{l}\text { Pro- } \\
\text { gramm } \\
\text { Rang }\end{array}$ & $\begin{array}{l}\text { Teil- } \\
\text { nehmer } \\
\text { Rang }\end{array}$ & $\begin{array}{l}\text { Tages- } \\
\text { zeitung } \\
\text { Rang }\end{array}$ & $\begin{array}{l}\text { Katholiken- } \\
\text { tagszeitung } \\
\text { Rang }\end{array}$ & $\begin{array}{l}\text { Fern- } \\
\text { sehen } \\
\text { Rang }\end{array}$ \\
\hline Kulturprogramme & 1 & 23 & 21,5 & 27 & 6 \\
\hline $\begin{array}{l}\text { Glaubensverkündigung, } \\
\text { Bekenntnis, Sakrament }\end{array}$ & 2 & 2 & 15 & 7 & 1 \\
\hline $\begin{array}{l}\text { Besinnung, Ruhe zum } \\
\text { Denken }\end{array}$ & 3,5 & 4 & 15 & 12,5 & 22 \\
\hline Begegnungen & 5 & 1 & 21,5 & 9,5 & 19 \\
\hline $\begin{array}{l}\text { Dritte Welt; Theologie der } \\
\text { Befreiung }\end{array}$ & 6 & 5,5 & 8 & 4 & 10 \\
\hline Bibelarbeit & 7 & 14,5 & 27,5 & 24 & 26 \\
\hline $\begin{array}{l}\text { Situation in der Kirche, Öku- } \\
\text { mene, aktuelle Probleme }\end{array}$ & 8,5 & 3 & 15 & 1,5 & 2,5 \\
\hline
\end{tabular}




$\begin{array}{lllll}\text { Pro- } & \text { Teil- } & \text { Tages- } & \text { Katholiken- } & \text { Fern- } \\ \text { gramm } & \text { nehmer } & \text { zeitung } & \begin{array}{l}\text { tagszeitung } \\ \text { sehen }\end{array} \\ \text { Rang } & \text { Rang } & \text { Rang } & \text { Rang } & \text { Rang }\end{array}$

Europa, -politik; Europäische

Einigung

Neue Technologien;

Umweltschutz

Frieden, Friedenspolitik

Kirchliche Institutionen

Soziale Marktwirtschaft,

Sicherheit; Arbeitslosigkeit

Lebenshilfe; Familie und

Erziehung

Soziale Probleme: Ausländer;

Randgruppen

Kirche und Jugend

Rolle der Frau in der Kirche

Verfolgte, bekennende Kir-

che; Menschenrechte

Schutz des ungeborenen

Lebens

Widerspruch zwischen Glaubenslehre und Glaubenspraxis

Politik der Partei die Grünen und Kirche

Motto des Katholikentags

(Exegese)

Organisation des Katholiken-

tags

Katholikentag, Bedeutung und Vergleich

„Katholikentag von unten “

Bestimmte Personen und

Gruppen auf dem Katholi-

kentag

Politische Persönlichkeiten auf dem Katholikentag**)

Kirchliche Würdenträger auf dem Katholikentag

Sonstige Inhalte

$\begin{array}{lllll}8,5 & 20,5 & 15 & 7 & 4,5\end{array}$

$\begin{array}{lllll}12,5 & 7 & 4 & 12,5 & 8\end{array}$

$\begin{array}{lllll}12,5 & 11,5 & 15 & 18,5 & 12,5\end{array}$

$\begin{array}{lllll}12,5 & 13 & 21,5 & 9,5 & 26\end{array}$

$\begin{array}{llllll}12,5 & 14,5 & 5,5 & 4 & 8\end{array}$

$\begin{array}{lllll}12,5 & 20,5 & 25,5 & 18,5 & 26\end{array}$

$\begin{array}{lllll}12,5 & 26,5 & 27,5 & 12,5 & 12,5\end{array}$

$\begin{array}{lllll}17 & 11,5 & 5,5 & 12,5 & 4,5\end{array}$

$\begin{array}{lllll}17 & 17,5 & 21,5 & 18,5 & 22\end{array}$

$\begin{array}{lllll}17 & 23 & 21,5 & 18,5 & 26\end{array}$

$\begin{array}{lllll}19,5 & 5,5 & 3 & 18,5 & 16\end{array}$

$\begin{array}{lllll}19,5 & 17,5 & 10 & 18,5 & 26\end{array}$

$\begin{array}{lllll}24,5 & 9 & 2 & 18,5 & 12,5\end{array}$

$\begin{array}{lllll}24,5 & 9 & 21,5 & 27 & 12,5\end{array}$

$\begin{array}{lllll}24,5 & 17,5 & 10 & 4 & 16\end{array}$

$\begin{array}{lllll}24,5 & 23 & 1 & 7 & 8\end{array}$

$\begin{array}{lllll}24,5 & 17,5 & 10 & 27 & 16\end{array}$

$\begin{array}{lllll}24,5 & 26,5 & 15 & 18,5 & 22\end{array}$

$\begin{array}{lllll}24,5 & 26,5 & 15 & 24 & 19\end{array}$

$\begin{array}{lllll}24,5 & 26,5 & 25,5 & 24 & 19 \\ 3,5 & 9 & 7 & 1,5 & 2,5\end{array}$

*) Vgl. Tabelle 1.

*) Ausgenommen Politiker der Partei Die Grünen. 
In der Beachtung von ausgewählten Inhalten ist das Fernsehen der „Katholikentagszeitung " näher als die zum Vergleich herangezogenen Tageszeitungen.

Die abschließende Frage, ob die Medien angemessen über den Katholikentag berichtet hätten, läßt sich global nach den vorliegenden Befunden nicht beantworten. Es wurde hinreichend deutlich, daß der Katholikentag ein äußerst komplexes Ereignis mit vielen Facetten war. Der Versuch, ihn in seiner vollen Breite zu erfassen, muß scheitern, da er zwangsläufig an der Oberfläche des Ereignisses bleiben wird. Er wurde auch von keinem der hier untersuchten Medien unternommen. Alle haben den Katholikentag punktuell erfaßt. Sie haben dabei unterschiedliche Aspekte herausgegriffen. Gemeinsam ist allen, daß sie in der Berichterstattung $z$ war einzelne Bereiche - vor allem inhaltliche - aufgegriffen haben, diese aber weitestgehend im Programm des Katholikentags verankert waren. Themen, die auf dem Katholikentag aufkamen, Ereignisse, die sich am Rande ereigneten, waren nicht bestimmend für das Gesamtbild der Berichterstattung.

Die Medien folgen in ihrem Verhalten insoweit den Teilnehmern des Katholikentags, da auch diese ihn nicht als ein einziges homogenes Ereignis erlebten. Auch für den Teilnehmer war der Katholikentag heterogen. Je weiter man den Besucher in statistische Gruppen „zerlegt“, desto mehr zerfällt eine vielleicht vermutete Gemeinsamkeit. Umgekehrt, je mehr verschiedene Medien man zur Analyse der Berichterstattung heranzieht, desto vielfältiger wird das Bild. Somit gab es für den Besucher nicht den Katholikentag, wie es auch nicht die Journalisten gab, die über den Katholikentag berichteten.

\section{Anmerkungen}

1 Vgl. Winfried Schulz: Die Konstruktion der Realität in den Nachrichtenmedien. Analyse der aktuellen Berichterstattung. Freiburg/München 1976.

2 Verschiedene Bereiche des Journalismus wurden bereits daraufhin untersucht. Für die Berichterstattung über kirchliche Ereignisse vgl. den nach Abschluß dieser Studie erschienenen Sammelband: Hans Maier, Otto B. Roegele, Manfred Spieker (Hrsg.): Katholikentage im Fernsehen. Referate der Arbeitstagung der Sektion für Politik- und Kommunikationswissenschaft bei der Jahresversammlung der Görres-Gesellschaft in Osnabrück am 7. Oktober 1985. Paderborn, München, Wien, Zürich 1987.

3 Die Untersuchung wurde im Auftrag des Westdeutschen Rundfunks, Köln, und des Zentralkomitees der deutschen Katholiken, Bonn, von dem Autor dieses Beitrags durchgeführt und die Ereignisse analysiert. Norbert Waldmann: Der Katholikentag in Aachen 1986. Perspektiven eines Ereignisses. Bd. 1: Textband, Bd. 2: Tabellen und Methoden, Hamburg 1987.

4 Ein ähnliches Vorgehen ist bei dieser Fragestellung auch in anderen Studien zu finden. Vgl. etwa James D. Halloran, Philip Elliott, Graham Murdock: Demonstrations and Communication: A Case Study. Harmondworth 1970.

5 Inhaltsanalysen klassifizieren - im Gegensatz zu subjektiv, impressionistischen Verfahren das zu untersuchende Material nach vorgegebenen Kategorien. Darauf aufbauend ist eine Quantifizierung und Analyse des Untersuchungsmaterials nach bestimmten Fragestellungen möglich. Vgl. etwa Bernhard Berelson: Content Analysis in Communication Research. New York 1952; Werner Früh: Inhaltsanalyse. Theorie und Praxis. MÜnchen 1981.

6 Die Welt, Frankfurter Allgemeine Zeitung, Frankfurter Rundschau, Süddeutsche Zeitung.

7 Für das Gesamtbild der Berichterstattung wäre es wünschenswert gewesen, auch die ZDFSendungen zu untersuchen. Letztlich war es die Entscheidung der Auftraggeber, beispielhaft für das Fernsehen, die WDR-Berichterstattung in die Untersuchung einzubeziehen. 
8 Herrn Dr. Uwe Magnus, Intendanz/Medienforschung des WDR, und Herrn Werner Hamerski, Programmgruppe Religion/Philosophie des WDR, sei an dieser Stelle für die Unterstützung gedankt.

9 Vgl. Klaus Schönbach: Trennung von Nachricht und Meinung. Empirische Untersuchung eines journalistischen Qualitätskriteriums. Freiburg/München 1977, S. 40.

$10 \mathrm{Vgl}$. Winfried Schulz, Rolf van Lessen, Cornelia Schlede, Norbert Waldmann: Die Bedeutung der audiovisuellen Berichterstattung für die Vermittlung politischer Einstellung. In AVforschung 15, 1976. S. 49-120.

11 In den Zeitungen wurde der Umfang in Spaltenzentimetern, beim Fernsehen in Sekunden festgehalten.

12 Die Befragung von Tagesgästen oder mehr oder weniger zufällig teilnehmenden Aachener Bürgern, hätte einen erheblichen Mehraufwand bei voraussichtlich ähnlichem Erkenntnisertrag und ebenfalls partieller Unwägbarkeiten hinsichtlich der Repräsentativität bedeutet. Herrn Dr. Vinzens M. Lissek vom Zentralkomitee der deutschen Katholiken und Herrn Lauber von der Geschäftsstelle des 89. Deutschen Katholikentags sei an dieser Stelle für ihre Unterstützung gedankt.

13 Die Rücklaufquote ist bei dieser Erhebung außerordentlich gut und gibt den erhobenen Daten eine große Aussagekraft. Vgl. Jürgen Friedrichs: Methoden empirischer Sozialforschung. Opladen 1982, S. 237.

14 Die gezogene Stichprobe war disproportional in Bezug auf den Anteil der Aachener Bevölkerung. Auch bei entsprechendem Redressement bleibt Nordrhein-Westfalen das Bundesland, aus dem die meisten Besucher kamen.

15 Pro Beitrag bzw. Informationseinheit, vgl. Anm. 11, wurde der Umfang in Spaltenzentimetern ermittelt.

\section{SUMMARY}

In a comparative analysis, the author examines the reporting of television and daily papers on the 1986 assembly of German Catholics in Aachen. He also includes in his judgement interviews of the participants and their impressions of the event.

\section{RESUME}

L'auteur examine dans une analyse parallèle, le compte-rendu des télévisions et quotidiens concernant „la journée catholique" 1986 à Aix-la-Chapelle. Il se rapporte aussi en cela à une interview des participants et à leur perception des évènements dans sa critique.

\section{RESUMEN}

Mediante un análisis comparativo el autor extudia las informaciones de la televisión y de los diarios sobre „El día de los católicos de 1986“ en Aquisgrán. Incluye en su critica también las preguntas a los participantes y sus impressiones respecto al acontecimiento. 